\title{
Sweetness and intensity of artificial sweeteners
}

HOWARD R. MOSKOWITZ ${ }^{2}$

HARVARD UNIVERSITY

In four experiments, groups of Os judged either the sweetness or the entire taste intensity of solutions of sucrose, cyclamate salts, cyclamate-saccharin mixtures, and sodium saccharin. The sensory functions obtained by magnitude estimation suggest that over the middle range of concentration the sweetness and intensity of the foregoing substances grow as power functions of concentration. As a first approximation, the exponents for sweetness and intensity are, respectively, 1.6 to 1.4 for sucrose, 1.0 to 0.8 for cyclamate salts, 0.6 to 0.85 for cyclamate-saccharin mixtures, and 0.3 to 0.6 for sodium saccharin.

Sugars grow in sweetness according to a power function of concentration, with a probable exponent of 1.3 (Moskowitz, 1968, 1970; Stevens, 1969). In log-log coordinates this relation between sweetness (S) and concentration (C) is expressed by the equation $\log S=1.3 \log \mathrm{C}+\log \mathrm{k}$. The intercept, $\mathrm{k}$, differs for different sugars and is a measure of relative sweetness at the concentration given by the intercept (Moskowitz, 1970). There are, however, many other sweeteners besides sugars. The present study concerns the sweetness functions for the artificial sweeteners, cyclamate and saccharin, whose quality of sweetness differs markedly from the sweetness produced by sugars. Usually the sweet taste of cyclamate and saccharin is accompanied by a bitter off-taste (Helgren et al, 1955) that increases with concentration. At the higher concentrations the bitter predominates and the artificial sweeteners no longer evoke the sweet taste.

In view of the presence of at least two distinct tastes in artificial sweeteners, it is important to separate the sweetness component from the rest of the taste. In the present study, functions both for sweetness and for total taste intensity were obtained for each of four sweetening substances: sucrose, sodium and calcium cyclamate, sodium and calcium Sucaryl (a mixture of cyclamate and saccharin), and sodium saccharin.

\section{PROCEDURE}

Stimulus solutions were prepared on a percent-by-weight basis (grams solute/grams solution) from reagent-grade chemicals (cyclamate salts and sodium saccharin) and from commercial brands (Domino cane sugar; "Sucaryl," "Ann Page Sweetener"). Solutions prepared with Cambridge tap water were made up 3 to 4 days prior to use. When not in use they were stored under refrigeration at $5^{\circ} \mathrm{C}$.

Different groups of $\mathrm{Os}$, chosen from a pool of 60 Harvard students, participated in the experiment. Each $O$ sat in front of a table on which were arranged the stimulus solutions in a linear array. Solutions were given in paper cups ( $3 / 4-\mathrm{oz}$ Sweetheart souffle cups), each containing 4 to $6 \mathrm{ml}$ of solution. The order of presentation was irregular and was changed for each $O$. In most of the experiments the $O$ sampled between 40 and 50 solutions, usually taking about $30 \mathrm{~min}$ to complete the session.

Prior to each session the $O$ was given the following written instructions: "In front of you is a series of solutions in paper cups. Your task is to tell how sweet/intense they seem by assigning numbers to them. If the second is nineteen times as sweet/intense as the first, then assign it a number nineteen times as large. If the second is one-eleventh as sweet/intense, then assign it a number one-eleventh as large, and so forth. Use fractions, decimals, and whole numbers, but make each assignment proportional to the sweetness/intensity as you perceive it. Rinse after each stimulus solution. If you cannot taste anything in the solution then rinse again and try the sample once more. If you still cannot taste anything, then use " 0 " for your magnitude estimate."

There was no time limit on the session. Each $O$ was free to proceed at his own pace. During the course of the sessions several Os complained about a bitter taste that remained after rinsing. In these cases they were instructed to rinse thoroughly, gargle, and wait a few seconds before proceeding.

\section{ANALYSIS}

The numerical matches (magnitude estimates) were analyzed with a local computer program called PSYCHOFIT (Panek \& Stevens, 1965). PSYCHOFIT provided geometric means, medians, and measures of variability (standard deviation and interquartile range) for the magnitude estimates of each stimulus concentration. In those instances in which Os reported " 0 " as their magnitude estimate the " 0 " was replaced by $0.1 \mathrm{X}$ the lowest magnitude estimate of each $O$. Thus each $O$ was given a different positive number corresponding to his estimate of " 0 ," with the property that it was the lowest of his numbers.

The geometric mean has been employed as the measure of central tendency for magnitude estimates because the logarithms of the judgments tend to be distributed symmetrically. In the present case the presence of " 0 " required that the median judgments be used instead, because the geometric mean of a set of numbers containing a zero becomes zero.

In all the experiments reported here each $O$ was left free to choose the modulus for his scale. As a result some individuals used numbers in the hundreds and thousands, whereas others used fractions. In order to reduce the magnitude estimates of all Os to a common modulus, without, however, changing the ratios of the numbers, a normalization procedure called modulus equalization was used (Lane, Catania, \& Stevens, 1961). The normalization required two steps. First, the responses of each $\mathrm{O}$ to a core set of six sucrose stimuli $(2 \%, 4 \%, 8 \%, 16 \%, 32 \%$, $64 \%$ ) were normalized by a single multiplier so that their geometric mean became 10.0. The ratios of magnitude estimates were left unchanged, but the size of the numbers was equalized for all Os. Second, the responses of each $O$ to the other stimulus concentrations in the same experiment were also multiplied by the same constant that had been used previously to normalize his response to sucrose. Again the ratios of magnitude estimates were left unchanged. The only change was in the absolute level of the magnitude estimates, and, for each $O$, that change was identical for all his numerical estimates. In each experiment every $O$ had a unique normalization multiplier, and when Os participated in two or more experiments they usually required two or more different multipliers. By this normalization two sweeteners that had been scaled in entirely different experiments, e.g., sodium saccharin and calcium cyclamate, could be compared to each other.

\section{RESULTS}

In Fig. 1 the logarithms of the median magnitude estimates of "sweetness" and "intensity" are plotted as a function of the 


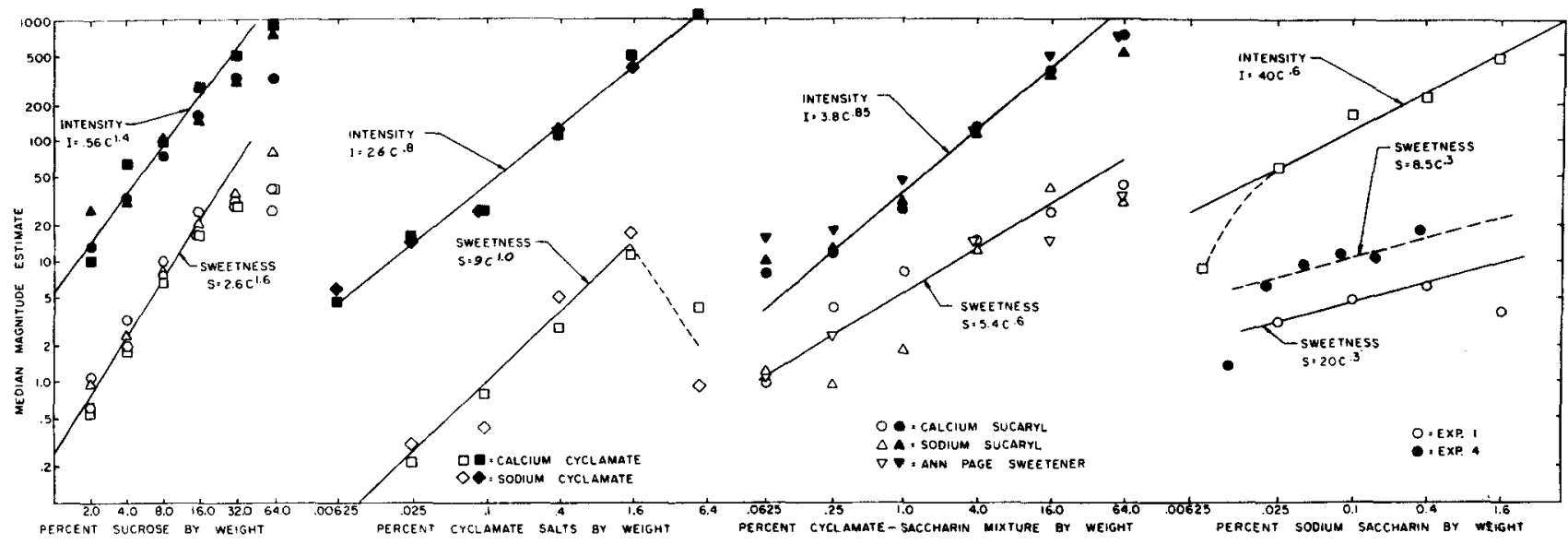

Fig. 1. Sweetness and intensity functions for four groups of sweet substances: sucrose, sodium and calcium cyclamate, cyclamatesaccharin ("Sucaryl") mixtures (sodium and calcium forms), and sodium saccharin. The coordinates are log-log in each case, so that straight lines suggest power functions.

logarithm of concentration. When sensory functions are linear in these logarithmic coordinates, they conform to power functions. In most cases the sweetness functions are linear only over a limited range of concentrations. In particular, the high concentrations of sucrose and the extreme concentrations (at both ends) of the artificial sweeteners systematically depart from a straight line. The straight lines in Fig. 1 were fitted only to the most linear segment of the function. Both the slope and the intercept were calculated by a least-squares procedure.

On the other hand, the functions that resulted when the Os were instructed to judge the overall intensity of the taste conform more nearly to power functions. The differences between the sweetness and the intensity functions become more pronounced as the exponents decrease in magnitude. In the case of sodium saccharin

Table 1

Conditions of Experiments

\begin{tabular}{|c|c|c|c|}
\hline Exp. & No. Os & Judgments/Os & Substance \\
\hline 1 & $\begin{array}{l}10 \\
10\end{array}$ & $\begin{array}{ll}2 & \text { Sweet } \\
2 & \text { Intensity }\end{array}$ & $\begin{array}{l}\text { Sucrose } \\
\mathrm{Na} \text { Cyclamate } \\
\mathrm{Ca} \text { Cyclamate }\end{array}$ \\
\hline 2 & $\begin{array}{l}10 \\
10\end{array}$ & $\begin{array}{l}2 \text { Sweet } \\
2 \text { Intensity }\end{array}$ & $\begin{array}{l}\text { Sucrose } \\
\text { Na Sucaryl* } \\
\text { Ca Sucaryl* } \\
\text { Ann Page* }\end{array}$ \\
\hline 3 & $\begin{array}{l}15 \\
15\end{array}$ & $\begin{array}{l}1 \text { Sweet } \\
1 \text { Intensity }\end{array}$ & $\begin{array}{l}\text { Sucrose } \\
\text { Na Saccharin }\end{array}$ \\
\hline 4 & 30 & I Sweet & $\begin{array}{l}\text { Sucrose } \\
\text { Na Saccharin }\end{array}$ \\
\hline
\end{tabular}

* Na Sucaryl is a commercial brand of sweetner, containing a 10:1 ratio of sodium cyclamate to sodium saccharin. Ca Sucaryl is a similar commercial brand containing a $10: 1$ ratio of calcum cyclamate to calcium saccharin. Both are sold by Abbott Laboratories, Inc., North Chicago, Ill. Ann Page is a 10:1 mixture of sodium cyclamate and sodium saccharin marketed by the A\& P Company. the difference between the functions is marked, and the sweetness exponent is approximately half of the intensity exponent.

The three simple sweetness functions (sucrose, cyclamate salts, sodium saccharin) differ from each other in slope (exponent). Sucrose grows most rapidly in sweetness with concentration, followed by the cyclamate salts, and finally by sodium saccharin. In the case of cyclamates the nature of the cation appears to make little difference in either the sweetness or the intensity function. There are two different sweetness functions for sodium saccharin, both with an exponent of approximately 0.3 , but with intercepts that differ by a factor of 2.5. Thus there are two separate estimates of the relative sweetness of sodium saccharin, differing by a factor of 2.5. On the other hand, the growth in sweetness for sodium saccharin is approximately the same in each experiment. The discrepancy may have resulted from different groups of Os, since the two functions were obtained in separate experiments. In general, the artificial sweeteners grow in sweetness less rapidly than sucrose, typically at a decelerating rate with concentration. At high concentrations the sweetness functions for the artificial sweeteners begin to decrease with increases in concentration, presumably because a bitter side-taste becomes predominant.

The functions for intensity are linear (i.e., power functions) throughout most of the range, except for the two highest concentrations of sucrose and the lowest concentration of saccharin. The slopes (exponents) for the intensities of cyclamate salts and Sucaryl mixtures are virtually identical, whereas the slopes for the sweetness of Sucaryl mixtures lie between the slopes of cyclamate and saccharin.

On the basis of the power-function exponents the sweeteners may be placed into three classes: sucrose, cyclamate salts, and saccharin. Sucaryl mixtures appear to be intermediate between the cyclamate salts and saccharin but resemble more closely the cyclamate salts, which predominate in Sucaryl by a factor of 10:1.

No single exponent accounts for either the sweetness or the intensity of the

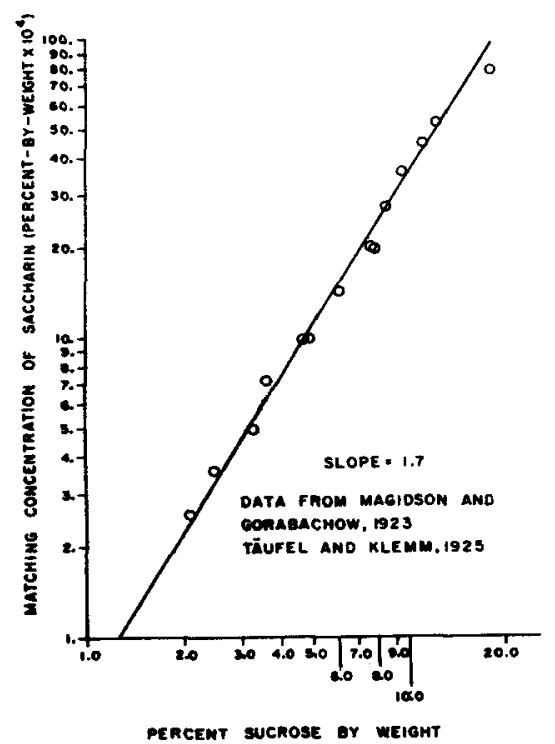

Fig. 2. Equal-sweetness functions traced out by direct matches between sucrose and saccharin sweetness (Magidson \& Gorabachow, 1923; Täufel \& Klemm, 1925). The coordinates are $\log -\log$. The slope (exponent) of the power function is approximately 1.7 . 
Fig. 3. Variability of magnitude estimates (interquartile range) expressed as decilogs (10ths of a logarithmic unit). In the region where the function is flat the variability is proportional to the mean judgment.

artificial sweetners. The functions suggest that there are probably two and possibly three classes of substances. One class (sugar) grows in sweetness at an accelerating rate (exponent greater than 1.0 ), whereas the other class grows at a decelerating rate (exponent less than 1.0), either mildly (cyclamates) or severely (sodium saccikarin).

Direct matches between the sweetness of sucrose and saccharin suggest that their exponents differ considerably. Two such matches were done in the 1920 s and reported in the German literature (Magidson \& Gorabacliow, 1923; Täufel \& Klemm, 1925). The equal-sweetness function (Fig. 2) iraced out by their results is linear in log-log coordinates, with a slope of approximately 1.7. If the exponent for sucrose is 1.3 , then the results of these direct matches suggest a probable saccharin exponent of 0.8 , rather than the value 0.3 found here. Stevens (1969) also suggests that the saccharin exponent is 0.8 , rather than 0.3 . The discrepancy in exponents may be due to differences in concentrations used. In both cases mentioned the concentrations are lower than those used here. Figure 1 suggests that there is a steep drop in the saccharin function at low concentrations, so that the function may actually be two-legged. The flatness of the function at high concentrations may result from the increase in the bitter side-taste with increases in concentration, an effect that would hinder the growth of sweetness. On the other hand, at low concentrations the bitter taste is faint, if present at all, and increases in concentration could produce concomitant increases in sweetness. The actual exponent for saccharin remains open for inquiry.

\section{Relative Sweetness of Sweeteners}

The relative sweetness of 15 sugars remained constant across concentrations, a convenient outcome of the finding that for the 15 sugars the sweetness function had an exponent of 1.3 (Moskowitz, 1970). One number, the intercept, sufficed as a measure of relative sweetness. In the present study the large differences found in the slopes of the four sweeteners preclude a single measure of relative sweetness. Instead, relative sweetness changes continuously with concentration.

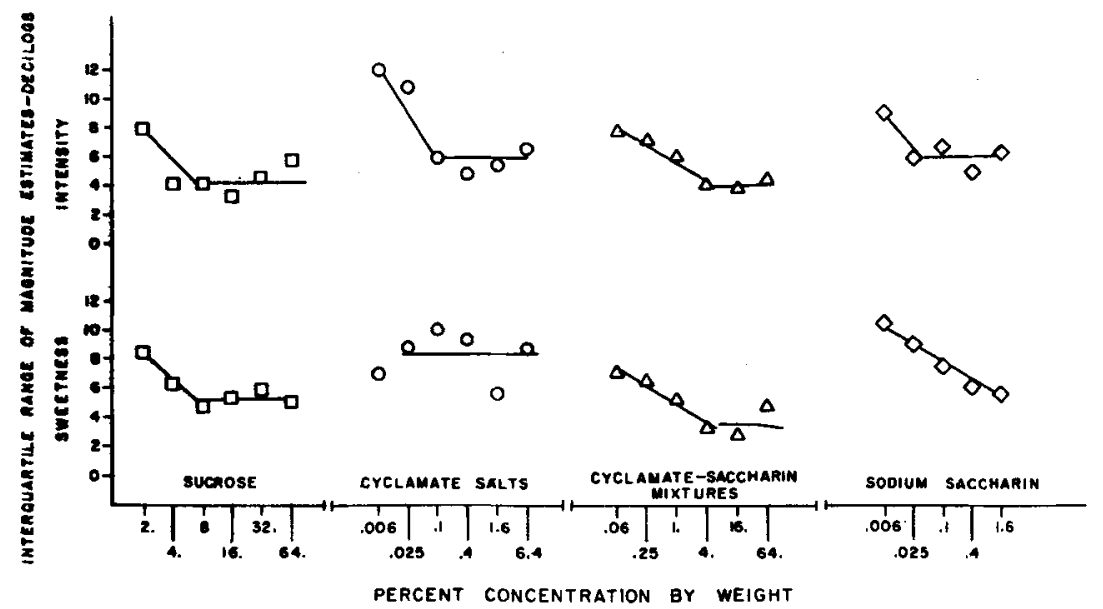

A simple graphical procedure may be used to calculate relative sweetness and equal-sweetness contours. For the sweetness of different substances to be compared at a single concentration, one need only find the median magnitude estimate given for those concentrations and then calculate the ratios. These ratios are estimates of relative sweetness at that concentration.

A graphic algorithm may also be used to obtain equal-sweetness functions. A straight line extended horizontally, at a particular sweetness level, will intersect each of the four sweetness functions. The physical concentrations corresponding to the points of intersection are those that are approximately equally sweet. Unfortunately, the graphic procedure must be used with extreme caution in the regions of stimulus concentrations beyond those used here. The behavior of the sweeteners at high concentrations suggests a decrease in sweetness, but the form of the decrease is as yet undetermined.

\section{Variability}

The variability of the magnitude estimates, both for the sweetness and for the intensity functions, behaves in a fashion consistent with Weber's law. Figure 3 presents the interquartile range of the magnitude estimates after the variability due to the scale sizes of individual $O s$ was removed by modulus equalization. The interquartile range was computed from the logarithms of the judgments, and it is plotted against the logarithm of concentration. The interquartile range decreases with concentration and usually becomes stable. Flat functions relating variability in logarithmic units to concentration suggest that variability is directly proportional to concentration.

\section{REFERENCES}

HELGREN, F. J., LYNCH, M. J., \& KIRCHMEYER, F. J. A taste panel study of the saccharin "off-taste." Journal of the American Pharmaceutical Association, 1955, 44, 353-355.

LANE, H. C., CATANIA, A. C., \& STEVENS, S. $S$. Voice level: Autophonic scale, perceived loudness, and effects of sidetone. Journal of the Acoustical Society of America, 1961, 33, 160-167.

MAGIDSON, O. J., \& GORABACHOW, S. W. Zur Frage der Süsigkeit des Saccharins. Das o-Benzoylsulfimid und seine elektrolytische Dissoziation. Chemische Berichte, 1923, 56B, 1810-1817.

MOSKOWITZ, H. R. Scales of intensity for single and compound tastes. Unpublished doctoral dissertation, Harvard University, Cambridge, Mass., 1968.

MOSKOWITZ, H. R. Ratio scales of sugar sweetness. Perception \& Psychophysics, 1970, $7,315-320$

PANEK, D. W., \& STEVENS, J. C. Psychofit, a computer program for the treatment of psychophysical data. Laboratory of Psychophysics, Harvard University, 1965, PPR 315.

STEVENS, S. S. Sensory scales of taste intensity. Perception \& Psychophysics, 1969, 6, 302-308.

TÄUFEL, K., \& KLEMM, B. Untersuchungen äber natürliche und künstliche Süsstoffe. I. Studien über den Süssungsgrad von Saccharin und Dulcin. Zeitschrift für Untersuchung der Lebensmittel, 1925, 50, 264-273.

\section{NOTES}

1. Research supported in part by a National Science Foundation predoctoral fellowship and in part by Grant NB-02974 from the National Institutes of Health. (Laboratory of Psychophysics Report PPR-356).

2. Current address: U.S. Army Natick Laboratories, Pioneering Research Laboratory, Natick, Massachusetts 01760.

3. The cyclamate salts and the sodium saccharin were provided by Abbott Laboratories, North Chicago, Illinois.

(Accepted for publication October 20, 1969.) 